\title{
A Novel Approach to Recommender System Based on
}

\section{Aspect-level Sentiment Analysis}

\author{
Yu Zhang ${ }^{1, ~ a ~}$, RuiFang Liü ${ }^{1, b}$, AoDong $\mathrm{Li}^{1, \mathrm{c}}$ \\ ${ }^{1}$ Beijing University of Posts \& Telecommunications, Beijing, 100876, China \\ accarmackz@live.com, 'Irf@bupt.edu.cn, cliaodong@bupt.edu.cn
}

Keywords: recommendation system; aspect-based sentiment analysis; opinion mining

\begin{abstract}
Traditional recommendation methods usually focus on utilizing user's features obtained from structured behavior information, which only contains coarse grained user interests. This paper presents a novel approach to introduce aspect-based sentiment analysis (or opinion mining) into recommender systems. By extracting aspects from the user's review text and determining the sentiment orientation of each aspect, we build the user and product model focusing on mining user's interests and the practical evaluations about the product. Experimental results show that the recommendation utilizing this model conduces to better performance than common traditional recommendation methods.
\end{abstract}

\section{Introduction}

Recommender systems, with an aim to provide personalized suggestions of products to the particular users, have been wildly used in practical applications [1]. Traditional recommendation methods usually focus on utilizing user profiles obtained from their past purchasing behaviors, from which the similarities [2] or relationships [3] between each user are generated. However, these structured data, such as purchasing behaviors or ratings range from 1 to 5 , only express the coarsest grained sentiment orientations that hardly reflect the details of user interests or his attitudes towards each part of the product. For example, a positive rating (4 or 5) about the entity does not mean that the author has positive opinions about all aspects of the entity. Likewise, a negative rating (1 or 2) document does not mean that the author is negative about everything.

Compared with the structured information, user review, as a natural language text in unstructured format, contains much finer grained sentiment orientations towards different aspects of a single item. To obtain the detail of author's opinion, we need to discover the aspects of this comment and determine whether the sentiment is positive or negative or the extent of positive/negative attitude on each aspect [4].

These opinions of various aspects of the product can be utilized to construct the user model as well as the product model. On the one hand, all unique aspects, which extracted from all comments made about different items by a particular user, form a list of aspects which opinion holder takes interested in. On the other hand, all comments about one particular product made by different people determine the general evaluation of this product. For example, here is a typical restaurant comment, "The ambiance is great and the stuff is friendly." The two aspects, ambiance and stuff, is definitely the aspects this user interested in, so that the customer would evaluates other restaurants on the same two aspects with a high probability. Meanwhile, the combination of aspects of various comments and their sentiment orientation (degree of positive or negative), to some extent, reflect a comprehensive assessment on different parts of this restaurant.

In this paper, we propose a novel approach to introduce aspect-based sentiment analysis into recommender systems. We extract aspect of the product using the topic model and then identify aspect-specific sentiment words using the SentiWordNet (a sentiment lexicon). We then use the result of sentiment analysis to make user interests model and the product model. By comparing two models of each user-product pair, we obtain the similarity of the user's interest and the product. A modification of traditional method based on this model conduces to much better performance. 


\section{Related work}

There have been a number of efforts focused on sentiment analysis. Pang et al. [5] first use supervised learning approaches to classify movie reviews into two classes. To extract the aspects of item and determine sentiment orientation of each aspect, Hu and Liu [6] propose a method aiming to discover aspects by finding frequent nouns and noun phrases. The occurrence of nouns and noun phrases (or groups), identified by a part-of-speech (POS) tagger, are counted and only the frequent ones are kept in their study. Popescu et al. [7] improve this method by removing the noun phrases that may not be aspects of entities using a pointwise mutual information (PMI) score. Such method works effectively in some situations. It doesn't consider the semantic similarity of each word however. In other words, this method cannot classify two similar word (e.g., atmosphere/ambience, staff/waiter) into one aspect class.

Similar to other natural language processing(NLP) problem, many supervised learning algorithms, such as hidden Markov models (HMM) and conditional random fields (CRF), have been proposed for this information extraction task [8]. Jin and Ho [9] applied a lexicalized HMM model to learn patterns to extract aspects and opinion expressions. Since these are supervised techniques, sufficient amount of labeled data for training is needed. To make things worse, which is different from other NLP tasks, one need manually annotate aspects and non-aspects in a corpus and make labeled data cover enough aspects to make sure it works well on complex aspect.

To solve the issues above, a series of methods using topic model have been proposed. Brody and Elhadad [10] proposed an unsupervised learning method using topic model to identify aspects and then identify aspect-specific sentiment words by considering adjectives only. In this paper, we improve this method by utilizing the sentiment lexicon to identify the aspect-specific sentiment words. This method doesn't acquire manually labeled data and works well on semantically similar words which means different words expressing the same or related property are automatically grouped together under the same aspect.

Zhang et al. [11] propose a user modeling method by evaluating the user's experience and skill. They then extract aspects of product and model the product with two sentiment orientation class of good or bad. They neglect the evaluations about the review by other user as well as the fine grained sentiment orientation of the opinion holder.

\section{Aspect Extraction}

We use latent Dirichlet allocation(LDA) model to discover the aspects in the whole document set. In order to infer the multiple aspects of a single review text, we regard each sentence as a separate document and run LDA model on these sentences. Thus we get the distribution of inferred aspects for each sentence in the data. Although in many actual situations, one sentence in a review probably contains two or more aspects, this LDA method still works well to find all aspects in the entire data and the words distribution of them. The core issue of LDA model is to determine the appropriate number of clusters. In this paper, we use a cluster validation procedure demonstrated by Niu in [12]. We run LDA model with the clusters numbers ranging from 5-30 and choose the model with the most consistent clustering by comparing each model.

To determine the representative words of each aspect, we compute a score, in the form of mutual information, for each noun in the document sets. The score is defined as follows:

$$
S_{a}(w)=p(w, a) \cdot \log \frac{p(w, a)}{p(w) \cdot p(a)}
$$

Where $p(w), p(a)$ are the probabilities of the word $w$ and the aspect a, and $p(w, a)$ is the probability of the word labeled with aspect. For each aspect, we select the top $\mathrm{k}_{\mathrm{a}}$ words of the score. The representative words set of each aspect intuitively show the performance of our aspects extraction process. Table I is a part of result from an experiment conducted using this algorithm on the Yelp's Academic Dataset. The aspect name is manually inferred by words in this cluster. We can see 
the LDA model works well on clustering relative words.

TABLE I. REPRESENTATIVE WORDS OF SOME ASPECTS

\begin{tabular}{|l|l|}
\hline \multicolumn{1}{|c|}{ Aspect } & \multicolumn{1}{c|}{ Representative words } \\
\hline atmosphere & atmosphere, ambience, wall, decor, experience \\
\hline staff & staff, wait, service, bill, cook, \\
\hline dessert & dessert, cream, chocolate, pancake, appetizer, drink \\
\hline portion & part, portion, size, full \\
\hline dinner & steak, seafood, salad, bread, wine \\
\hline
\end{tabular}

To utilize each review text, we need determine which aspect each word denotes. We compute $p(\mathrm{a} \mid \mathrm{w})$ of each word and choose the aspect with the highest conditional probability as the aspect denoted by the word.

\section{Sentiment Orientation}

Since the aspects have been extracted from the whole review dataset, we then determine the sentiment orientation of each aspect of a review. For each word in documents, With the help of SentiWordNet, we can get a positive, negative, and objective score and the three scores add up to 1. We define a word as sentiment word if objective score less than 0.3(or other threshold).

For each word in a review text, if it is in any aspect's representative words set, we identify its closest sentiment word. If the word has no sentiment word nearby, we regard the sentiment upon this word is neutral and its sentiment contribution to this aspect is zero. Assume we extract $\mathrm{n}$ aspects from the whole dataset and aspect $a_{i}$ (consider these word has sentiment word nearby only) occurs $m$ times in a review. Then we represent a review as a n-dimensional vector $\left(a_{1}, a_{2}, \ldots, a_{n}\right)$ in which each dimension represents an aspect. We compute $a_{i}$ for the aspect $i$ as follows:

$$
a_{i}=\frac{1}{m} \sum_{j=1}^{m} \frac{\left(p s_{j}-n s_{j}\right) \cdot \max \left(p s_{j}, n_{j}\right)}{\left|p s_{j}-n s_{j}\right| \cdot\left(p s_{j}+n s_{j}\right)} \text {. }
$$

Where the function $\max (\mathrm{a}, \mathrm{b})$ denotes the maximum of $\mathrm{a}$ and $\mathrm{b}, \mathrm{ps}_{\mathrm{j}}$, $\mathrm{ns}_{\mathrm{j}}$ denote the positive score and negative score obtained from SentiWordNet. Thus we transfer the unstructured natural language text into a structured vector representation. Each dimension of $\mathrm{r}$ represents a specific aspect and its value indicate the extent of the sentiment orientation of the aspect. As can be seen from the equation, the value denotes the opinion holder's general attitude towards this aspect. Note that the sign of the value reflects the polarity of the sentiment orientation.

\section{Product Modeling}

A product gets a number of reviews. Since the sentiment orientations of each aspect is determined, we indicate the product as a vector by make a weighted summation of all reviews. Websites providing opinion services often provide an evaluation feature by witch other users could judge this review whether it is useful or not. Thus we allocate each review a weight calculated from the number of other users' useful votes.

$$
\overrightarrow{\mathrm{p}}=\sum_{\mathrm{i}=1}^{\mathrm{k}} \frac{\mathrm{n}\left(\mathrm{ack}_{\mathrm{i}}\right)}{\sum_{\mathrm{i}=1}^{\mathrm{k}} \mathrm{n}\left(\mathrm{ack}_{\mathrm{i}}\right)} \overrightarrow{\mathrm{r}}_{\mathrm{i}}
$$

Where $\mathrm{k}$ is the number of reviews, ack $\mathrm{k}_{\mathrm{i}}$ is the number of useful acknowledgement (set 1 if ack is 0$) r_{i}$ received and the function $n(x)$ is a nonlinear function. The Nonlinear normalization process 
of $\mathrm{ack}_{\mathrm{i}}$ is critical because ack $\mathrm{i}_{\mathrm{i}}$ of each review ranges widely. Specifically, most people tend to interact with hot reviews, however, those reviews with few acknowledgements still have certain utilization values. Using equation above, we make the model of each product and transfer it into a vector $p$.

\section{User Modeling}

A customer makes reviews about many products and the aspects extracted from his reviews reflect his interests in the same class of products. In other words, the occurrence frequency in review text of a specific aspect indicates the user's concern about this property. Thus we make the user model by calculating occurrence frequency of each aspect in a similar way above and represent the user as a vector $u=\left(\mathrm{u}_{1}, \mathrm{u}_{2}, \ldots, \mathrm{u}_{\mathrm{i}}, \ldots\right)$ in which each element is calculated as below.

$$
\mathrm{u}_{\mathrm{i}}=\frac{\mathrm{n}\left(\mathrm{of}_{\mathrm{i}}\right)}{\sum_{\mathrm{i}=1}^{\mathrm{n}} \mathrm{n}\left(\mathrm{of}_{\mathrm{i}}\right)}
$$

Where of $\mathrm{f}_{\mathrm{i}}$ is the occurrence of the aspect, $\mathrm{n}(\mathrm{x})$ is a nonlinear function. Thus we have the user model $u$. For those aspect doesn't appear in the review text, we define $u_{i}$ as average value of others.

\section{Recommendation}

For each pair of candidate product $\mathrm{i}$ and user $\mathrm{j}$, we compute a score by simply make the dot product of the two vector.

$$
\text { Score }(\mathrm{i}, \mathrm{j})=\overrightarrow{\mathrm{p}_{\mathrm{i}}} \cdot \overrightarrow{\mathrm{u}_{\mathrm{j}}}
$$

Where vector $p_{i}$ is the product vector and vector $u_{j}$ is the user vector. This score can be regarded as a Rocchio-like similarity. $p_{i}$ has both positive and negative elements. A positive one mean the product owns positive overall reputation on this aspect so that the part score is high if the user has an interest in this aspect. If one aspect of this product has bad reputation, people with more concern about this aspect would suffer more from it than those who don't take interested in it. Based on this idea, we make recommendation to user by calculating the score of user-product pair and selecting the product with top k scores.

\section{Experiment}

We test our approach as well as other traditional recommendation methods on Yelp's Academic Dataset. The dataset contains more than 60,000 local business of various kinds and more than 30,000 users. We choose all restaurants those have more than 5 review as the products. And choose all user who have more than 5 reviews to form the user dataset. Consider the dataset of local businesses has a significant trait different with other dataset (e.g., movie reviews) that the purchasing behavior is much sparser, we make recommendation and evaluate it in a range of local region. We test our approach, collaborative filtering based on matrix factorization and popularity-based recommendation as ranking models. We utilize mean reciprocal rank(MRR) to evaluate these three methods. MRR is the average of the reciprocal ranks of results for the test data. Assume rank $k_{i}$ denotes the rank of product with highest rating score in suggestion list and MRR is given by:

$$
\operatorname{MRR}=\frac{1}{\mathrm{~m}} \sum_{\mathrm{i}=1}^{\mathrm{m}} \frac{1}{\operatorname{rank}_{\mathrm{i}}}
$$


TABLE II .PERFORMANCE COMPARISION

\begin{tabular}{|c|c|}
\hline \multicolumn{1}{|c|}{ Method } & MRR \\
\hline popularity-based & 0.289 \\
\hline collaborative filtering & 0.531 \\
\hline opinion-mining-based & 0.627 \\
\hline
\end{tabular}

Table II shows the recommendation method based on opinion-mining have a better performance than traditional collaborative filtering based on matrix factorization in the situation of small scale and accuracy demanded.

\section{Conclusion}

In this paper we propose a novel approach to recommender system by introducing aspect-based sentiment analysis into user and product modeling. Experimental results show it has better performance than common recommendation methods such as collaborative filtering. This approach of opinion mining could deeply mine the interests of customer as well as restore the reputation of each product aspect due to reviews it gets. Consider the technics for aspect-level sentiment analysis are still developing and far from ideal, this approach has much room for improvement.

Although the fine grained sentiment analysis makes user modeling more personalized, this method show weakness in searching candidate product in a large scale. Predictably, further work on hybrid recommend systems combined with aspect-level sentiment analysis and main recommendation approaches is promising.

\section{References}

[1] BURKE, Robin. Hybrid recommender systems: Survey and experiments. User modeling and user-adapted interaction, 2002, 12.4: 331-370.

[2] PAZZANI, Michael J.; BILLSUS, Daniel. Content-based recommendation systems. In: The adaptive web. Springer Berlin Heidelberg, 2007. p. 325-341.

[3] DONG, Yuxiao, et al. Link prediction and recommendation across heterogeneous social networks. In: Data Mining (ICDM), 2012 IEEE 12th International Conference on. IEEE, 2012. p. 181-190.

[4] LIU, Bing. Sentiment analysis and opinion mining. Synthesis Lectures on Human Language Technologies, 2012, 5.1: 1-167.

[5] PANG, Bo; LEE, Lillian; VAITHYANATHAN, Shivakumar. Thumbs up?: sentiment classification using machine learning techniques. In: Proceedings of the ACL-02 conference on Empirical methods in natural language processing-Volume 10. Association for Computational Linguistics, 2002. p. 79-86.

[6] HU, Minqing; LIU, Bing. Mining and summarizing customer reviews. In:Proceedings of the tenth ACM SIGKDD international conference on Knowledge discovery and data mining. ACM, 2004. p. 168-177.

[7] POPESCU, Ana-Maria; ETZIONI, Orena. Extracting product features and opinions from reviews. In: Natural language processing and text mining. Springer London, 2007. p. 9-28.

[8] HOBBS, Jerry R.; RILOFF, Ellen. Information extraction. Handbook of natural language processing, 2010, 2.,

[9] JIN, Wei; HO, Hung Hay; SRIHARI, Rohini K. A novel lexicalized HMM-based learning framework for web opinion mining. In: Proceedings of the 26th Annual International Conference on Machine Learning. 2009. p. 465-472. 
[10] BRODY, Samuel; ELHADAD, Noemie. An unsupervised aspect-sentiment model for online reviews. In: Human Language Technologies: The 2010 Annual Conference of the North American Chapter of the Association for Computational Linguistics. Association for Computational Linguistics, 2010. p. 804-812.

[11] ACIAR, Silvana, et al. Informed recommender: Basing recommendations on consumer product reviews. Intelligent Systems, IEEE, 2007, 22.3: 39-47.

[12] NIU, Zheng-Yu; JI, Dong-Hong; TAN, Chew-Lim. I2r: Three systems for word sense discrimination, chinese word sense disambiguation, and english word sense disambiguation. In: Proceedings of the 4th International Workshop on Semantic Evaluations. Association for Computational Linguistics, 2007. p. 177-182. 